coronary artery diameter was measured by quantitative angiography. An incremental pacing protocol that raised heart rate to a maximum of $150 \mathrm{bpm}$ was undertaken in all patients via a temporary right atrial pacing wire. Pacing was performed in the presence of saline vehicle and then either L-NMMA or SMTC (one inhibitor per patient; $\mathrm{n}=9$ each group).

Results SMTC $(0.625 \mu \mathrm{mol} / \mathrm{min})$ and L-NMMA $(25 \mu \mathrm{mol} / \mathrm{min})$ both reduced basal CBF to a similar extent $(-22.8 \% \pm 1.24 \%$ vs $-26.8 \% \pm 2.16 \% ; n=9$ each; $p=N S$ ). During saline infusion, $C B F$ increased with atrial pacing from $58.7 \pm 9.90$ to $87.4 \pm 17.3 \mathrm{ml} / \mathrm{min}$ $(\mathrm{n}=9, \mathrm{p}<0.05)$. During L-NMMA, the increase in CBF was significantly blunted compared to that during saline $(\triangle \mathrm{CBF} 17.7 \pm 4.54 \mathrm{ml} /$ $\min$ vs $28.7 \pm 8.08 \mathrm{ml} / \mathrm{min}$ during saline; $\mathrm{n}=9, \mathrm{p}<0.05$ by 2 -way ANOVA). In patients receiving SMTC, however, the increase in CBF with pacing was similar to that during saline $(\Delta \mathrm{CBF} 36.5 \pm 6.05 \mathrm{ml} /$ $\min$ vs $25.2 \pm 6.65 \mathrm{ml} / \mathrm{min}$ during saline; $\mathrm{n}=9, \mathrm{p}=\mathrm{NS}$ by 2 -way ANOVA). SMTC and L-NMMA both reduced basal coronary artery diameter to a similar extent $(\mathrm{n}=9)$. L-NMMA blunted the pacinginduced increase in coronary artery diameter $(n=9, p<0.05$ vs saline vehicle) whereas SMTC had no effect ( $n=9, p=N S)$.

Conclusion These results suggest that increases in human coronary blood flow in response to incremental atrial pacing are mediated by eNOS-derived NO rather than nNOS-derived NO.

Funding Supported by the British Heart Foundation.

\section{REMOTE ISCHAEMIC PRECONDITIONING AND HUMAN ATRIAL TRABECULAE IN THE DIABETIC HEART}

doi:10.1136/heartjnl-2012-301877b.107

${ }^{1} \mathrm{R}$ Sirohi, ${ }^{1} \mathrm{~L}$ Candilio, ${ }^{1} \mathrm{G}$ Babu, ${ }^{2} \mathrm{~N}$ Roberts, ${ }^{2} \mathrm{D}$ Lawrence, ${ }^{2} \mathrm{~A}$ Sheik, ${ }^{2} \mathrm{~S}$ Kolvekar, ${ }^{2} \mathrm{~J}$ Yap, 'D J Hausenloy, 'D M Yellon. ${ }^{1}$ The Hatter Cardiovascular Institute, London, UK; ${ }^{2}$ The Heart Hospital, University College London Hospital, London, UK

Introduction Remote ischaemic preconditioning (RIPC) using brief cycles of upper or lower limb ischaemia and reperfusion has been reported to protect the heart against ischaemia-reperfusion injury (IRI). Previous studies suggest that the diabetic heart is more resistant to the cardioprotective effects of myocardial ischaemic preconditioning. Whether the diabetic heart is amenable to RIPC is unknown and is investigated in this study.

Methods Non-diabetic and diabetic patients undergoing elective coronary artery bypass graft $(\mathrm{CABG})$ surgery were randomised to receive three different treatment protocols after the induction of anaesthesia: (1) Control-no RIPC; (2) RIPC1 comprising 3-five min cycles of upper arm cuff inflation/deflation; or (3) RIPC2 comprising 2 -five min cycles of simultaneous upper and lower limb cuff inflations/deflations (total 4). A section of the right atrial appendage was harvested, from which atrial trabeculae were isolated and subjected to 90 min simulated ischaemia and 120 min simulated reperfusion, at the end of which the recovery of baseline contractile function was determined.

Results Atrial trabeculae harvested from diabetic ( $N=13$ patients) and non-diabetic control patients ( $\mathrm{N}=20$ patients) were demonstrated to recover $24.5 \% \pm 2.4 \%$ and $29.3 \% \pm 1.3 \%$ of baseline contractile function, respectively. Prior treatment of patients with RIPC1 increased the recovery of function in both non-diabetic $(50.4 \% \pm 1.9 \% ; \quad \mathrm{p}<0.05)$ and diabetic $(41.6 \% \pm 1.9 \% ; \quad \mathrm{p}<0.05)$ patients. Interestingly, the stronger RIPC2 stimulus resulted in a greater recovery of function in both non-diabetic $(59.3 \% \pm 1.9 \%$; $\mathrm{p}<0.05)$ and diabetic $(50.7 \% \pm 2.1 \% ; \mathrm{p}<0.05)$ patients. As a positive control direct hypoxic preconditioning (HPC) of atrial trabeculae also improved the recovery of function $(56.4 \% \pm 1.8 \%$ with HPC vs $27.5 \% \pm 1.7 \%$ in control; $\mathrm{N}=10$ patients; $\mathrm{p}<0.05)$. The administration of the MEK-Erk1/2 inhibitors U0126 and PD98059 at the onset of reperfusion abrogated the protective effect in both nondiabetic $(30.9 \% \pm 0.8 \%$ U0126 and $31.3 \% \pm 0.8 \%$ PD98059; $p>0.05)$ and diabetic $(28.6 \% \pm 0.9 \%$ U0126 and $30.0 \% \pm 1.2 \%$ PD98059; $\mathrm{p}>0.05)$ atrial trabeculae.

Conclusion We demonstrate for the first time that in vivo RIPC can protect ex vivo atrial trabeculae against simulated IRI. Both nondiabetic and diabetic atrial trabeculae were amenable to RIPC protection. Increasing the intensity of the RIPC stimulus resulted in greater functional recovery. The pro-survival kinase MEK-Erk1/2 appears to contribute to RIPC protection in human atria trabeculae.

\section{REMOTE ISCHAEMIC CONDITIONING IS IMPAIRED IN DIABETES}

doi:10.1136/heartjnl-2012-301877b.108

S A Edroos, ${ }^{*}$ A P Vanezis, M J Davies, N J Samani, G C Rodrigo. Department of Cardiovascular Sciences, University of Leicester, Leicester, UK

Aim Remote conditioning, whereby intermittent non-critical ischaemia of remote peripheral muscle can protect cardiac muscle from ischaemia-reperfusion (I-R) injury even when applied after the onset of cardiac ischaemia, has emerged as a potential therapeutic manoeuvre for reducing I-R injury. Whether such protection is mediated via serum factors and whether the development of the protection is impaired in disease states is unclear. We sought to develop a cell based model for assessing I-R injury, and utilised this to screen sera from both control healthy volunteers and type 1 diabetic patients for blood borne cardioprotective signals capable of mediating remote conditioning protection.

Methods Subjects studied included healthy volunteers and patients with type 1 diabetes with microvascular complications (retinopathy, microalbuminuria). Control (unconditioned) blood samples were taken prior to remote conditioning. An upper limb was then occluded with a tourniquet [ $30 \mathrm{~mm} \mathrm{Hg}$ suprasystolic, $5 \mathrm{~min}$ ] and released to reperfuse the arm [5 $\mathrm{min}$ ] three times. Conditioned blood was collected immediately afterwards from the contralateral arm. Serum was separated and stored at $-80^{\circ} \mathrm{C}$ until assay. Serum was screened for its protective capacity using a cellular model of I-R. Myocardial ischaemia was simulated by centrifugation of freshly isolated rat cardiac ventricular myocytes into a pellet [ $30 \mathrm{~min}$ ]. Gaseous diffusion was prevented by an impermeant layer of mineral oil. Reperfusion injury was simulated through dispersal of the pellet in oxygenated saline solution. Cell viability was determined by propidium iodide staining for necrosis and calcein-AM counterstaining for viability.

Results In healthy subjects $(\mathrm{n}=21)$ unconditioned serum resulted in necrosis of $23.7 \% \pm 4.9 \%$ (mean \pm SEM) of cells. Conditioned serum resulted in a significant reduction of necrosis to $6.4 \% \pm 5.1 \%$ $(p<0.05)$. However conditioned serum from diabetics showed no evidence of protection compared with unconditioned diabetic serum $(51.1 \% \pm 4.6 \%$ vs $45.7 \% \pm 6.4 \%$ respectively, $n=14, p>0.05$, NS). The difference in necrosis rates between conditioned serum from healthy subjects and diabetics was highly significant $(p<0.001)$. Results were similar whether the serum was applied before centrifugation of the cells (mimicking preconditioning in vivo) or on reperfusion (mimicking postconditioning).

Conclusion We report the development of a novel bioassay that can be used to quantitatively assess the strength of the serum-based signal that mediates remote conditioning. We further show that this signal is significantly impaired in patients with advanced diabetes. The later finding has important implications for the interpretation of clinical trials of remote conditioning, which include diabetics.

Funding Funded by the Leicester NIHR Cardiovascular Biomedical Research Unit. 